\title{
Teaching Kitchen Research Conference November 11-12, 2020
}

\author{
David M. Eisenberg, MD
}

\begin{abstract}
Editor's Note: Give 'em an inch and they'll take a mile. The phrase comes to mind in reflecting on the actual reach of a movement, nominally about food, that is on display in the "teaching kitchens" that are gaining stature nationally and globally in hospitals, corporations, and other institutional settings. The first paragraphs of this commentary from David Eisenberg, MD, the founder of the Teaching Kitchens Collaborative, introduce the multi-faceted "culture of health" that these institutional healing agents promote. JACM is again honored to partner with Eisenberg and the Teaching Kitchen Research Conference to publish the abstracts of their 2020 meeting. Eisenberg invites you into these emerging outposts of whole health then guides you through highlights of the meeting, and of the scientific presentations. Read on and you will likely agree that my framing at the top of this note wasn't quite right. Better to say: Give 'em an inch and they'll give you miles of life better and more healthily lived.
\end{abstract}

—John Weeks, Editor-in-Chief

$\mathbf{T}$ EACHING KITCHENS, AS currently designed and implemented across the United States and globally, have been designed as learning environments that include a kitchen; however, they are more than culinary instruction sites.

Teaching kitchens typically include instruction in most, if not all, of the following: (1) nutrition education, specifically what to eat more of or less of and why; (2) hands-on culinary instruction, that is, basic cooking skills for the home cook; (3) information about physical activity and its critical importance in health optimization; (4) mindfulness and its critical relationship to diet, portion control, satiety, and resilience; (5) the judicious use of web-based resources and information technologies; (6) the relationship between food choices and the environment; and (7) motivational interviewing strategies and health coaching techniques whereby personal motivations can be identified and leveraged to successfully achieve sustained behavioral change. ${ }^{1}$

Emerging teaching kitchen models serve as in-person or virtual life skills learning laboratories intended to promote health and wellness, and as clinical research centers whereby the impact of diet and lifestyle change on a range of behavioral, metabolic, genetic, clinical, and financial variables can be rigorously evaluated.

The conceptual origins of teaching kitchens include two philosophical tenets from both ancient China and ancient Greece:

1. Prevention is always superior to intervention. ${ }^{2}$ In The Yellow Emperor's Classic of Internal Medicine, ${ }^{2}$ attributed to the Legendary Yellow Emperor-who was believed to have lived from $2700 \mathrm{BC}$ to $2600 \mathrm{BC}$-this tenant is conveyed poetically as follows: "To admin- ister medicine to diseases which have already developed and thereby suppress bodily chaos, which has already occurred, is comparable to the behavior of those who would begin to dig a well after they had grown thirsty or those who would begin to cast weapons after they have engaged in battle. Would these actions not be too late.",

2. The way we eat, move, and think impacts our health, and determines our recuperative capacity. ${ }^{2}$

In addition, both Chinese and Greek medical thought leaders of the 5th century B.C. appreciated that one's "constitution," as referenced by Hippocrates, and one's "ancestral Qi", also known as "vital energy" in Chinese theory, are also determinants of one's health and resilience. Accordingly, modern teaching kitchens can be viewed as "learning laboratories" and "translational research centers" where the relationships between food, health, our environment, and the regulation of our genes (and microbiota) can be rigorously studied.

The first ever Teaching Kitchen Research Conference (TKRC) was held in February 2018, ${ }^{3}$ hosted by the Harvard T. H. Chan School of Public Health (Harvard Chan School) in association with the Teaching Kitchen Collaborative (www .teachingkitchens.org), an invitational network of $>30$ institutions with teaching kitchens dedicated to the development of best practices and the creation of a research network whereby teaching kitchens can be systematically evaluated, refined, and scaled for maximum impact (Appendix A1).

The second TKRC recently took place November 1112, 2020. Due to generous support from a grant from the National Institutes of Health (NIH; R13AT010554-01 from 
the National Center for Complementary and Integrative Health) and from unrestricted grants from the Meshewa Farm Foundation and the Campus for Health, LTD (Japan), the conference had the resources to provide free registration to anyone interested in participating. In addition, this conference was formally endorsed by both the Academic Consortium for Integrative Medicine and Health and by the American College of Lifestyle Medicine.

While the 2018 in-person TKRC was held in California and attracted 125 registrants from the United States and a small number of registrants from abroad, ${ }^{3}$ this second TKRC was held virtually due to the COVID-19 pandemic and attracted $>2500$ registrants from 79 countries. More than 900 registrants confirmed the existence of teaching kitchens within their respective institutions. This highlights how teaching kitchens are becoming more ubiquitous and are being designed and implemented for educational and research purposes across the international community. These figures also demonstrate that the desire to share "best practices," novel educational models, and emerging research findings within and across teaching kitchens has increased considerably since 2018 .

The 2020 TKRC included presentations from five speakers in addition to 24 oral abstract presentations, 37 poster presentations, and 2 panel discussions. ${ }^{4}$ The panel discussions focused on (1) methods currently in place to use teaching kitchens to educate health care professionals and (2) research challenges and opportunities involving the evaluation of teaching kitchens during and after the COVID-19 pandemic. Importantly, each keynote presentation was recorded and can be publicly viewed on demand (please visit https://www.tkresearchconference.org/recordings for more information).

Highlights of the TKRC welcome and keynote presentations, and a selection of oral abstracts, are briefly summarized hereunder.

David Eisenberg, director of Culinary Nutrition at the Harvard T.H. Chan School of Public Health and principal investigator of this NIH-sponsored research conference, provided a welcome and overview of the field. He discussed the development of teaching kitchens in the context of the annual educational conference, "Healthy Kitchens, Healthy Lives" (www.healthykitchens.org) cosponsored by the Harvard T. H. Chan School and the Culinary Institute of America and offered to health care professionals since 2006; the launch of the Teaching Kitchen Collaborative (www .teachingkitchens.org), an invitational network of organizations with teaching kitchens committed to building best practices and to the evaluation of teaching kitchen interventions with respect to changes in behaviors, clinical outcomes, and costs; and the launch of a new "Food is Medicine Map" (https://teachingkitchens.org/map-fim) being built for the purpose of identifying and showcasing teaching kitchen programs, as well as other "Food Is Medicine" programs, nationally and globally. Dr. Eisenberg also described current challenges in light of the COVID-19 pandemic and ongoing efforts by Teaching Kitchen Collaborative members to pivot from in-person instruction to virtual teaching platforms. Lastly, Dr. Eisenberg shared thoughts about the design, use, and evaluation of teaching kitchens of the future in both the working and learning environments. ${ }^{1}$
Frank Hu, chair of the Department of Nutrition at the Harvard T. H. Chan School of Public Health, provided a keynote presentation on "The Role of Teaching Kitchens in Precision Nutrition Research and Practice.' After describing the recently published 2020-2030 Strategic Plan for $\mathrm{NIH}$ Nutrition Research, ${ }^{5}$ Dr. Hu provided an overview of the field of precision nutrition, including advances in genomics and other omics technologies. Dr. Hu proposed that teaching kitchens can be used as (1) precision nutrition research laboratories, (2) educational venues for current and future nutrition professionals, (3) natural experimental settings to evaluate diet and lifestyle effects across teaching kitchen venues and populations, (4) instructional classrooms to translate research findings into practice, and (5) platforms for promoting sustainability to a global audience.

Richard Rothstein, chair of the Department of Medicine at the Dartmouth Geisel School of Medicine, provided a plenary presentation entitled: "Teaching Kitchens in Academic Health Systems: Superfluous, Advocated or Essential?" Professor Rothstein discussed the need for changes in medical education and training with regard to competencies relating to nutrition information and advice. He conveyed that teaching kitchens, functioning as learning laboratories, have demonstrated clinically meaningful outcome improvements, that evidence is accumulating for their incorporation into future health care systems, and that future research in this area will not only lead to scientific discovery and define the impact of teaching kitchens, but also provide the necessary financial capital to build and sustain such programs in the future. He discussed the role of teaching kitchens in the emerging "Food is Medicine" movement, the role teaching kitchens can play in reshaping the food landscape within hospitals and health systems for patients and communities, and mentioned the additional value of teaching kitchens as powerful "antidotes" to burnout among health care providers. Teaching kitchens, Dr. Rothstein argued, can improve the quality and reduce the costs of health care.

Nicole Farmer, research scientist at the NIH Clinical Center, presented a plenary entitled, "Translational Precision Nutrition: Teaching Kitchens and Precision Nutrition of the Future.' She conveyed that precision nutrition research, across the NIH and elsewhere, should include but not be limited to the study of genomics and omic-related technologies, but rather also include research involving cooking and other relevant self-care behaviors. She added that such research must simultaneously contend with the relevance of social determinants of health on self-care behaviors and outcomes, and should investigate how teaching kitchens can and should be studied in the context of research aimed at reducing health disparities, especially during and after the current COVID-19 pandemic.

Ruthie Schwab, Global Food Program Manager, Google, Inc., and Zoe Schweitzer, Global User Enablement Program Manager, Compass at Google, co-presented a plenary entitled, "Google as a Case Study for Corporate Food Education." They described "Food at Google" and the role of teaching kitchens over the past several years and more recently in the context of COVID-19, as well as "learnings" from this past year. They described how teaching kitchens have been used to enable Google employees to engage, to convene, to learn about, and co-create food; strengthen teams and foster connections; and to create unique experiences that 
inspire deeper interest in food system-related values and communities. In addition, they described ongoing efforts to pivot from in-person to digital teaching kitchen classes and to develop new "food at home" sites specifically for their large employee population. They also shared Google's evolving metrics whereby the performance of Google's Teaching Kitchens will be measured over time.

A number of original scientific abstracts included in the TKRC are noteworthy. These include:

- The abstract by Berquist et al. (original abstract [OA]1.01) describes the evaluation of Emory University's "Healthy Kitchen Collaborative's MultiDisciplinary Teaching Kitchen Self-Care Curriculum." In this study, benefits-eligible Emory employees $(n=37)$ were offered a multi-disciplinary teaching kitchen educational intervention in the form of five 4hour classes. This teaching kitchen model was feasible and effective as a strategy to increase health-promoting behaviors among an employee population.

- The abstract by Luchsinger et al. (OA2.04) describes a "Collaborative Enhanced Nutrition Care Program for Adults with Serious and Persistent Mental Illness" at the Kalamazoo Valley Community College. This program, which integrates culinary medicine into behavioral health services and includes the incorporation of culinary training using a mobile kitchen unit, was found to have multiple positive impacts on subjects $(n=27)$ with serious and persistent mental illness. As an editorial note, this study suggests the need for future studies involving teaching kitchens as applied to a range of patient populations treated by behavioral, mental health, and rehabilitation specialists.

- The abstract by LaCroix et al. (OA4.02) describes "Baseline Knowledge, Habits and Confidence of the Veterans Health Administration (VHA) Healthy Teaching Kitchen Participants." Data from 1373 participants from 36 VHA Healthy Teaching Kitchen sites suggest that most U.S. veterans who participate in teaching kitchen programs are older males with obesity who do not meet the recommended daily requirements with regard to vegetable, fruit, bean, and nut consumption, and who could benefit from enhanced meal planning and teaching kitchen-related instruction.

- The abstract by Jacobs et al. (OA4.03) described "Innovative Food Procurement Guidelines" applied to 10 hospitals within the Northwell Health System, the largest health system in the Greater New York region. These novel "healthy quality food initiatives," based on explicit Northwell Health food procurement guidelines, were initiated in June 2016. Between 2016 and 2020, Press Gainey Scores relating to the question: "How would you rate the quality of your food" rose from 9\% (2016) to 56\% (2019); total food purchases of $\$ 35$ million at baseline rose by only 3\% (identical to the cost of living increase over this same period); standards were raised with regard to the purchase of meats and fish; and a range of healthier — and well received—food items, including healthier (non-prefried) potatoes, artisanal (whole grain) breads, and healthier desserts were incorporated into standard hospital dining programs.
The authors conclude "It is possible to apply evidencebased food procurement policies to improve nutritional value and quality of hospital-based foods while increasing customer/patient satisfaction without significant increases in food expenditures."

- The abstract by Steinberg et al. (OA4.04) entitled, "Evaluating the Impact of Kaiser Permanente's Thrive Kitchen Culinary Medicine Program on Healthy Eating and Cooking Confidence and Behaviors," describes changes in cooking confidence and health-related behaviors among 362 participants who attended teaching kitchen classes at the mobile teaching kitchen at Kaiser Permanente, San Francisco, between 2017 and 2019. The majority found these classes to be useful, learned new skills, gained confidence, and were inspired to improve their eating and cooking habits.

- The abstract by Westfall et al. (OA5.03) entitled, "Teaching Kitchen Improves Health Professional Students' Nutrition Knowledge, Perceived Culinary Skills, and Confidence Providing Nutritional Counseling" describes a study in which UCLA students of medicine, nursing, dentistry, and public health were recruited through e-mail to participate in a "Teaching Kitchen Intervention." Based on pre-post data from 85 participants, this educational intervention resulted in significant improvement in students' nutrition knowledge, culinary skills, and confidence-providing nutritional counseling.

- Additional abstracts offer a sense of the diversity of teaching kitchen applications being evaluated in 2020 and include abstracts describing teaching kitchen programs in:

(1) A publicly funded city library (the Philadelphia Free Library Culinary Literacy Center) where teaching kitchen programs are offered to new immigrants using teaching kitchen classes to learn English as a second language, to teach cooking skills to adults with disabilities, and to teach nutrition facts and culinary skills to inner city youth and others (i.e., $>6200$ students per year in 2019) (poster abstract [P]-4.08);

(2) an OB/GYN practice in Boston (at Brigham and Women's Hospital) for pregnant women with a history of gestational diabetes (OA2.02);

(3) an RD training program at the University of Texas School of Public Health that requires evidence of competencies involving both culinary instruction and instruction in the design and implementation of family home vegetable gardens (P-5.10);

(4) a public Botanical Garden (Phipps Botanical Gardens in Pittsburgh) whose teaching kitchen provides information on nutrition basics, culinary instruction, and Motivational Interviewing to University of Pittsburgh medical students, as well as $>1000$ adults and 400 children per year (OA3.02); and

(5) a program in Alberta, Canada, where a teaching kitchen program has been successfully customized for senior citizens in an effort to enhance health behaviors and quality of life (P-1.02).

- An additional abstract by Massa et al. (OA 4.01) entitled, "Global Estimates of Cooking Frequency Prior to 
COVID-19" summarizes results from a Gallup Poll survey of 152,974 adults across 142 countries around the world with respect to cooking frequency. Data are based on a novel series of questions (Cooking Frequency Questionnaire) that was embedded within the Gallup Poll Survey in 2019, to assess the frequency of "cooking from scratch," and provides information on cooking frequency variability worldwide, gender disparities in cooking frequency, and the relationship between cooking frequency and life evaluation and positive experiences. This research provides a novel replicable measure of "cooking from scratch" and global baseline data regarding cooking frequencies, by country, pre- COVID-19.

Lastly, subject matter experts facilitated eight breakout sessions. These included discussions with respect to teaching kitchens as applied to (1) precision nutrition; (2) international collaborations; (3) integrative and lifestyle medicine; (4) educating health professionals; (5) clinical practice and research; (6) agriculture and sustainable food systems; (7) inter-professional synergies of registered dietitian-nutritionists, chefs, mind-body, and other teaching kitchen instructors; and (8) challenges and opportunities relating to enhancing health equity. The goal of each breakout was to identify (1) topics that are missing from the current list of teaching kitchen research priorities, (2) organizations and thought leaders who are currently missing from this multi-disciplinary line of inquiry, and (3) collaborations that do not yet exist but should be explored and nurtured to advance research involving teaching kitchens worldwide.

Our shared circumstances in the year 2020 as related to the COVID-19 pandemic; rising rates of noncommunicable chronic disease (e.g., obesity and diabetes); global climate change; and systemic racism informed many aspects of this scientific meeting. Most agreed that we will not-and cannot - return to "normal life pre-COVID 19," but rather, will need to collectively "build back better" in the years ahead. This conference made a strong case for the inclusion of teaching kitchens in the development of cocreated future plans and research opportunities intended to encourage a culture of health. Moreover, the involvement of so many individuals and organizations with teaching kitchens from around the globe suggests that there will need to be more TKRCs in the years ahead.

\section{Author Disclosure Statement}

The author declared the following potential conflicts of interest with respect to the research, authorship, and/or publication of this article: David Eisenberg, MD, is the executive director of the Teaching Kitchen Collaborative; co-director of the Harvard-Culinary Institute of America Healthy Kitchens, Healthy Lives educational conference; scientific adviser to Campus for Health, LTD, Japan; scientific adviser to Cookpad, Inc., Japan; scientific adviser to Better Therapeutics, Inc.; and a member of the Health and Wellness Advisory Board of Barilla, Inc., Italy.

\section{References}

1. Eisenberg DM, Imamura A. Teaching kitchens in the learning and work environments: The future is now. Global Adv Health Med 2020 [Epub ahead of print]; DOI: 10.1177/ 2164956120962442.

2. Veith I. The Yellow Emperor's Classic of Internal Medicine. Berkeley, CA: University of California Press, 2015.

3. Eisenberg DM. Teaching Kitchen Collaborative Research Day February 7, 2018. J Altern Complement Med 2018;24: 615-618.

4. Teaching Kitchen Research Conference. Abstracts. J Altern Complement Med 2020;26:A1-A24.

5. National Institutes of Health [homepage on the Internet]. 2020-2030 Strategic Plan for NIH Nutrition Research A Report of the NIH Nutrition Research Task Force. 2020. Online document at: https://www.niddk.nih.gov/about-niddk/ strategic-plans-reports/strategic-plan-nih-nutrition-research, accessed October 30, 2020.

Address correspondence to:

David M. Eisenberg, MD

Department of Nutrition

Harvard T.H. Chan School of Public Health 665 Huntington Avenue, Building 2 Boston, MA 02115

USA

E-mail: deisenbe@hsph.harvard.edu 


\section{Appendix A1. Teaching Kitchen Collaborative (www.teachingkitchens.org) Member Organizations 2020-2021}

\section{Member Organizations}

- 1440 Multiversity

- Alberta Health Services/University of Calgary

- Bon Secours Richmond Health System (beginning 2021)

- Boston Medical Center

- Cabell Huntington Hospital-Huntington Kitchen/ Marshall University

- Children's Healthcare of Atlanta

- Cleveland Clinic (Center for Lifestyle Medicine)

- University of Colorado Anschutz Health and Wellness Center (beginning 2021)

- Dartmouth-Hitchcock Culinary Medical Program

- FamilyCook Productions

- Free Library of Philadelphia (Culinary Literacy Center)

- Hackensack Meridian Health

- Kaiser Permanente San Francisco Medical Center

- MaineGeneral Health (Prevention and Healthy Living)

- MedStar Health, Washington, DC

- Northeastern University (Dining Services)

- Northwell Health System

- Northwestern University (Osher Center for Integrative Medicine)

- Palo Alto Medical Foundation

- Phipps Conservatory (beginning 2021)

- Presbyterian Community Health (beginning 2021)

- Providence Milwaukie Hospital

- Stanford University (Residential \& Dining Enterprises, School of Medicine \& Stanford Prevention Research Center)

- University of Texas Health School of Public Health, Nourish Program

- Turner Farm, Inc., in collaboration with University of Cincinnati (Academic Health Center \& College of Medicine, Center for Integrative Health and Wellness)

- University of California, Berkeley (College of Natural Resources; Health Services)

- University of California, Los Angeles (Chancellor Block's Healthy Campus Initiative)
- University of Michigan (Health System, MHealthy)

- University of Minnesota (Center for Spirituality \& Healing)

- University of Southern California (Keck School of Medicine)

- University of Vermont Medical Center

- Veterans Health Administration (VHA) Healthy Teaching Kitchen (HTK) Program

- YMCA of Greater Pittsburgh-Sampson Family Branch

\section{Member Grantors}

- Barilla and Barilla Center for Food \& Nutrition (BCFN) Foundation

- Campus for Health, Inc. (Japan)

- Compass Group, North America

- Google, Inc.,

\section{Supporting Foundations}

Principal support provided by:

- Sampson Foundation

- Peter Alfond Foundation

Additional support provided by:

- Meshewa Farm Foundation

\section{TKC Leadership}

David Eisenberg, MD, Executive Director Christine Hamann, MBA, MPH, Director

Emily Baron, Coordinator

\section{Contact}

info@teachingkitchens.org 